Take the Fourier sine transform of Eq. (12) and then eliminate the derivatives of $u$ by integrating by parts. This gives

$$
\begin{aligned}
w(\xi, t)=\int_{0}^{\infty} u(x, t)\left[a\left(b-h \kappa \xi^{2}\right) \sin \xi x+\xi(a+b\right. & \left.\left.-h_{\kappa} \xi^{2}\right) \cos \xi x\right] d x \\
& +a h_{\kappa} \xi u(0, t)-h_{\kappa \xi} \frac{\partial u(0, t)}{\partial x} .
\end{aligned}
$$

Let

$$
\phi(\xi)=\int_{0}^{\infty} f(x)\left[a\left(b-h \kappa \xi^{2}\right) \sin \xi x+\xi\left(a+b-h \kappa \xi^{2}\right) \cos \xi x\right] d x
$$

Then

$$
w(\xi, 0)=\phi(\xi)+a h \kappa \xi f(0)-h \kappa \xi f^{\prime}(0)
$$

So

$$
w(\xi, s)=\frac{\phi(\xi)+\kappa \xi a b g(s)+\kappa \xi a h V}{s+\kappa \xi^{2}}
$$

Hence

$$
w(\xi, t)=[\phi(\xi)+\kappa \xi a h V] \exp \left(-\kappa \xi^{2} t\right)+\kappa \xi a b \int_{0}^{t} g(\tau) \exp \left[-\kappa \xi^{2}(t-\tau)\right] d \tau .
$$

But

$$
w(x, t)=\frac{2}{\pi} \int_{0}^{\infty} w(\xi, t) \sin \xi x d \xi
$$

Therefore, from Eqs. (12) and (23),

$$
u(x, t)=\frac{2}{\pi} \int_{0}^{\infty} w(\xi, t) \frac{a\left(b-h_{\kappa} \xi^{2}\right) \sin \xi x+\xi\left(a+b-h_{\kappa} \xi^{2}\right) \cos \xi x}{a^{2}\left(b-h_{\kappa} \xi^{2}\right)^{2}+\xi^{2}\left(a+b-h_{\kappa} \xi^{2}\right)^{2}} d \xi,
$$

where $w(\xi, t)$ is given by Eq. (22), and $\phi(\xi)$ by Eq. (19). From Eq. (3) and (24) one may obtain $v(t)$.

\title{
NOTE ON THE SYMMETRICAL PROPERTY OF THE THERMAL CONDUCTIVITY TENSOR*
}

\section{By M. LESSEN (University of Pennsylvania)}

According to the Onsager reciprocal hypothesis, the thermal conductivity tensor among other similar properties of matter is symmetrical. In the following note it is demonstrated that for some cases of interest, the property of symmetry according to the Onsager hypothesis is not a necessary piece of information.

Assuming a space filled with a continuous medium at temperature $T$, having a

*Received June 15, 1955. 
thermal conductivity of $K_{i i}$ such that the conducted flow of heat through an elemental area $d s$ having associated direction cosines $l_{i}$ is

$$
-\left(K_{i j} T_{, i}\right) l_{i} d s .
$$

The net rate of heat flow into a volume bounded by a closed surface " $s$ " then is

$$
\int_{s}\left(K_{i j} T_{, i}\right) l_{i} d s
$$

The surface integral may be transformed to a volume integral,

$$
\int_{.}\left(K_{i i} T_{, i}\right) l_{i} d s=\int_{0}\left(K_{i j} T_{, i}\right)_{, i} d v .
$$

It is therefore necessary to investigate the properties of the form

$$
\left(K_{i j} T_{, j}\right)_{, i} \text {. }
$$

Considering the case where

$$
\begin{gathered}
K_{i i}=K_{i i}(T), \\
\left(K_{i j} T_{, i}\right)_{, i}=K_{i i} T_{, i i}+\frac{d K_{i i}}{d T} T_{, i} T_{, i}
\end{gathered}
$$

$T_{, i i}$ and $T_{, i} T_{, j}$ are both symmetrical in $i$ and $j$, therefore only the symmetrical portion of $K_{i j}$ will matter in $\left(K_{i j} T_{, i}\right)_{, i}$.

Since the case of $K_{i i}=K_{i j}(T)$ applies to a large class of practical applications, it is important to note, that for this case the anti-symmetrical portion of $K_{i j}$ if it existed at all would not contribute to a first law of thermodynamic energy accounting.

\section{A CONVERSE TO THE VIRTUAL WORK THEOREM FOR DEFORMABLE SOLIDS*}

BY W. S. DORN (Aircraft Gas Turbine Development Department, General Electric Company) AND

A. SCHILD (Carnegie Institute of Technology)

1. Introduction. Consider a continuous body occupying a volume $V$ and bounded by a closed surface $S .{ }^{1}$ Any system of stresses $\sigma_{i i},{ }^{2}$ satisfying the equilibrium conditions for zero body forces

$$
\begin{aligned}
& \sigma_{i i, i}=0, \\
& \sigma_{i j}=\sigma_{i i},
\end{aligned}
$$

${ }^{*}$ Received July 27, 1955. A. Schild's participation was supported by a research grant from the National Science Foundation.

'It is assumed that the body is simply connected and that the surface $S$ is composed of a finite number of pieces of each possessing a continuously turning tangent plane. All of the functions will be assumed to possess as many continuous derivatives in $V$ and on $S$ as are necessary for the theorems which will be used later.

2The subscripts range over the values $1,2,3$ and repeated subscripts will be summed over the entire range. Subscripts following a comma denote partial differentiation with respect to Cartesian coordinates $x_{i}$, e.g., $\sigma_{i, i}=\partial \sigma_{i j} / \partial x_{i}$. 